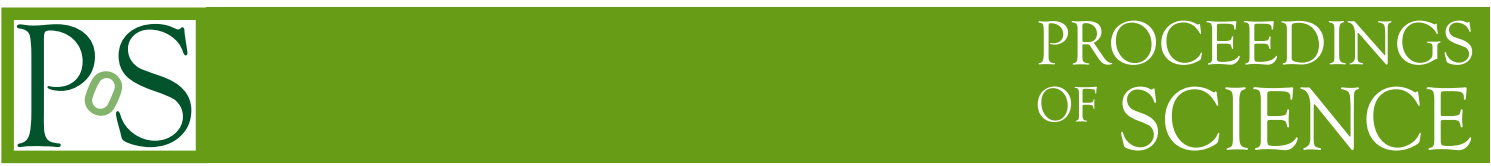

\title{
Construction of the Belle II TOP counter
}

\author{
K. Matsuoka*, For the Belle II TOP group \\ KMI, Nagoya University \\ E-mail: matsuoka@hepl.phys.nagoya-u.ac.jp
}

The Belle II experiment operating at SuperKEKB of the luminosity frontier accelerator will perform high precision flavor physics measurements. An efficient particle identification is essential for Belle II to extend its reach toward new physics, and it is realized by the TOP counter. The TOP counter is a novel ring imaging Cherenkov detector, which measures the time of propagation (TOP) of the internally reflected Cherenkov photons. The TOP counter mainly consists of a quartz optics, micro-channel-plate photomultiplier tubes and front-end electronics. After the long $R \& D$ of more than a decade, we succeeded in producing those components, assembling the 16 TOP counter modules and finally installing them in 2016. That is one of the biggest milestones of the Belle II construction. This article reviews the TOP counter in terms of the production of each component and the construction.

The 3rd International Symposium on "Quest for the Origin of Particles and the Universe" 5-7 January 2017

Nagoya University, Japan

${ }^{*}$ Speaker. 


\section{Introduction}

After the successful B-factory experiments [1], which confirmed Kobayashi-Maskawa theory with about $1 \mathrm{ab}^{-1}$ data, the Belle II experiment [2] is going to search for new physics via precision measurements with $50 \mathrm{ab}^{-1}$ data to be collected at the next generation B-factory, SuperKEKB [3]. In Belle II $K^{ \pm} / \pi^{ \pm}$particle identification in the barrel region is done by 16 modules of the TOP (Time-Of-Propagation) counter [4-7] illustrated in Fig. 1. The expected misidentification probability of pions, for example, is $3 \%$ when one selects $1-2 \mathrm{GeV} / \mathrm{c}$ kaons at $86 \%$ efficiency, while it was about $10 \%$ in Belle [8].

The TOP counter is a state-of-the-art Cherenkov ring imaging detector, which mainly consists of a $2.7 \mathrm{~m}$ long quartz optics, photon sensors at the end of the optics and front-end electronics attached to the photon sensors as shown in Fig. 1. It identifies kaons and pions by means of precise timing measurement of internally reflected Cherenkov photons in the quartz optics as well as the time of flight of the particle. The time of propagation of those photons depends on the Cherenkov angle or the mass of the particle of a given momentum by the inner tracker. One of the key techniques for the TOP counter is to propagate the "ring" image without distortion by the quartz optics. In addition the photon sensors and the electronics are required to have an excellent time resolution better than $50 \mathrm{ps}$, which is essential to distinguish the time difference between Cherenkov photons from a kaon and a pion. Detecting the photons with a high efficiency is also essential because the typical number of detected photons per track is only about 20. Hence we developed a square-shaped micro-channel-plate photomultiplier tube (MCP-PMT) [9-14] which can be arrayed to have a large photocoverage.

It was quite challenging to incorporate those features. After the TOP counter concept was proposed at Nagoya in 2000, it took a long time to prove the principle of the TOP counter using a full-scale prototype at a beam test $[15,16]$ in 2013 and to get the construction of all the 16 and a spare TOP counter modules done in 2016. This article reviews the TOP counter in terms of the production of the key components and the construction.
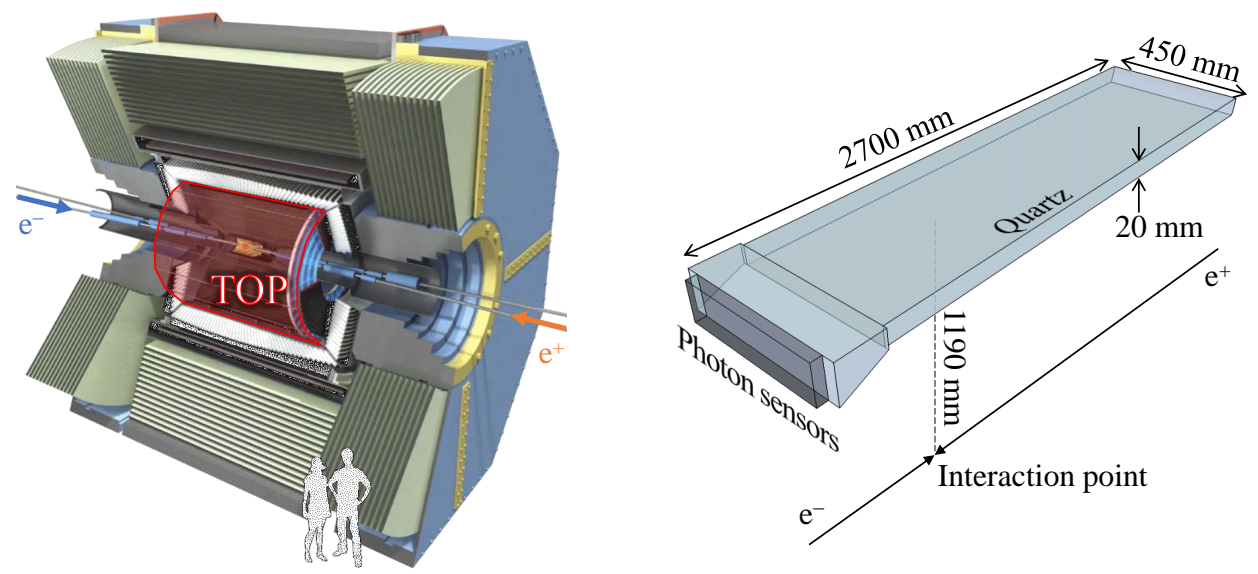

Figure 1: Schematic view of the Belle II detector indicating the barrel region covered by 16 modules of the TOP counter (left) and one of the TOP counter modules (right). 
Table 1: Specifications of the quartz bar. The flatness, perpendicularity, parallelism and roughness in this table indicate the specification for the largest surfaces.

\begin{tabular}{ll}
\hline Length & $1250 \pm 0.50 \mathrm{~mm}$ \\
Width & $450 \pm 0.15 \mathrm{~mm}$ \\
Thickness & $20 \pm 0.10 \mathrm{~mm}$ \\
Flatness & $<6.3 \mu \mathrm{m}$ \\
Perpendicularity & $<20 \operatorname{arcsec}$ \\
Parallelism & $<4 \operatorname{arcsec}$ \\
Roughness & $<5 \AA$ (RMS) \\
\hline
\end{tabular}

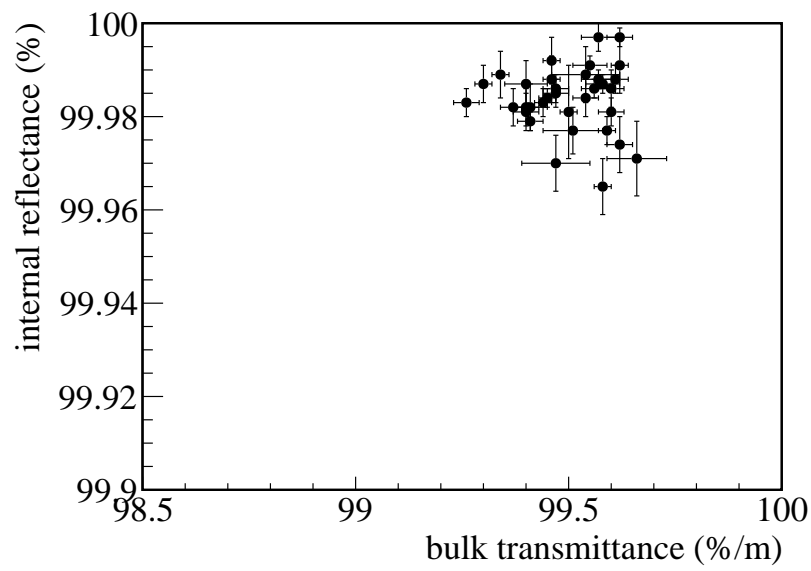

Figure 2: Measured internal reflectance and bulk transmittance of the quartz bars.

\section{Key components of the TOP counter}

\subsection{Quartz bar}

The quartz optics consists of two $1250 \times 450 \times 20 \mathrm{~mm}$ bars, which form a $2500 \mathrm{~mm}$ long bar, a prism at the end of the bar and a mirror at the other end. The material of all of the optics is Corning 7980 synthetic fused silica, which has no inclusions and is free of striae. To keep the Cherenkov image after typically a few hundreds of reflections in the quartz bar, the surfaces of the quartz bar are highly polished. The requirements for the surfaces is stringent as listed in Table 1. The preproduction and production of 34 quartz bars including two spares started in 2012 and 2014, respectively. Zygo Corporation produced thirty quartz bars and Okamoto Optics Works, Inc. produced four including two spares. For quality assurance the internal reflectance and the bulk transmittance of the produced bars were measured by using a laser. As shown in Fig. 2 all the quartz bars met the requirements which were $>99.9 \%$ internal reflectance and $>98.5 \% / \mathrm{m}$ bulk transmittance.

\subsection{MCP-PMT}

The MCP-PMT (Fig. 3) was developed in collaboration with Hamamatsu Photonics K.K. It contains two micro-channel-plates, of which channel diameter and thickness are $10 \mu \mathrm{m}$ and $400 \mu \mathrm{m}$, respectively. It has an enough gain to detect single photons even in $1.5 \mathrm{~T}$ of the Belle II solenoid. Because the electrons are multiplied inside the very narrow channels of the thin plate, the MCP-PMT has a transit time spread less than $40 \mathrm{ps}$ for single photons. The outer size of the MCP-PMT is $27.6 \times 27.6 \mathrm{~mm}^{2}$, and the size of the photocathode is $23 \times 23 \mathrm{~mm}^{2}$. Thus $73 \%$ of the prism edge face can be covered by the photocathode of two rows of 16 MCP-PMTs. A multi-alkali ( $\mathrm{NaKSbCs)}$ photocathode is adopted to fulfill the required quantum efficiency which is at least $24 \%$ and $28 \%$ on average of all MCP-PMTs at the peak wavelength around $360 \mathrm{~nm}$.

The 512 MCP-PMTs to be installed in 16 TOP counter modules and more than 60 spares were produced in five years from 2011. The measured peak quantum efficiency for the 512 MCP-PMTs 


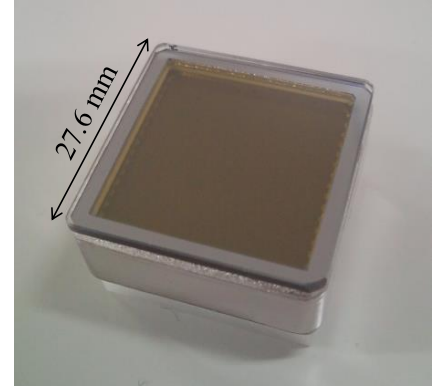

Figure 3: MCP-PMT for the TOP counter.

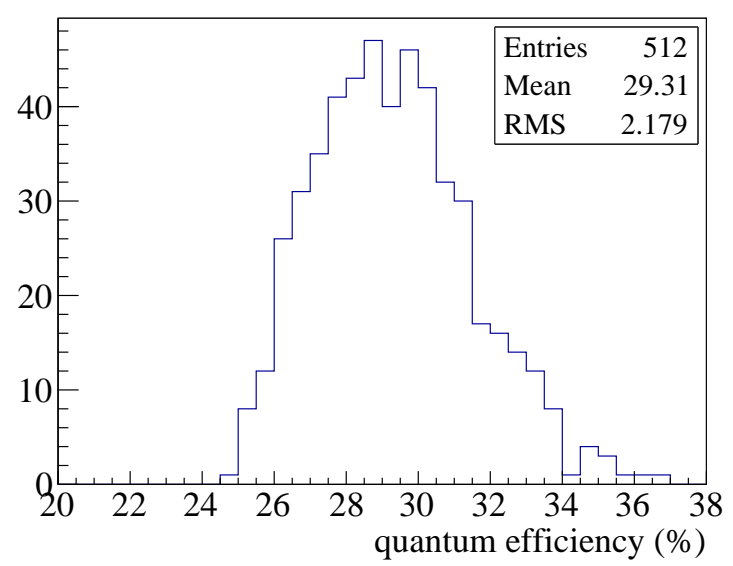

Figure 4: Measured quantum efficiency at a peak wavelength around $360 \mathrm{~nm}$ for the installed 512 MCP-PMTs.

is plotted in Fig. 4. The achieved average quantum efficiency is $29.3 \%$, which is greater than the requirement.

Another big achievement in terms of the MCP-PMT R\&D is extension of the lifetime. The lifetime of the MCP-PMT is limited by deterioration of the photocathode or the degradation of the quantum efficiency due to outgassing from the micro-channel-plates induced by the multiplied electrons. The quantum efficiency degrades as a function of the integrated amount of the output charge per photocathode area, which is dominated by the severe beam background in Belle II. It is estimated by a Monte Carlo simulation to be several $\mathrm{C} / \mathrm{cm}^{2}$ after collecting $50 \mathrm{ab}^{-1}$ data at the MCP-PMT gain of $5 \times 10^{5}$. On the other hand the lifetime when the quantum efficiency drops down to $80 \%$ of the beginning was $0.1 \mathrm{C} / \mathrm{cm}^{2}$ or less for the early prototypes of the MCP-PMT [13]. It was improved to $1.1 \mathrm{C} / \mathrm{cm}^{2}$ on average of 12 samples of the first production version, $10.5 \mathrm{C} / \mathrm{cm}^{2}$ on average of 8 samples of the second version, and at least $13.6 \mathrm{C} / \mathrm{cm}^{2}$ for 8 samples of the third version [17].

\subsection{Front-end electronics}

A eight-channel, multi-giga sample per second, transient waveform recorder ASIC (ApplicationSpecific Integrated Circuit) [18] was developed for the TOP counter readout by the University of Hawaii. Four ASICs are mounted on a carrier board with an FPGA (Field Programmable Gate Array) which controls the ASICs and processes the data. Four carrier boards are stacked with another board which is a master control, and they compose a board stack of dense and compact package to fit in the limited space behind the MCP-PMT array.

\section{Construction of the TOP counter}

\subsection{Assembly of the TOP counter}

Assembly of the 16 production TOP counter modules started in March 2015 and finished in April 2016 almost as planned. The assembly of each module starts with alignment and gluing of 

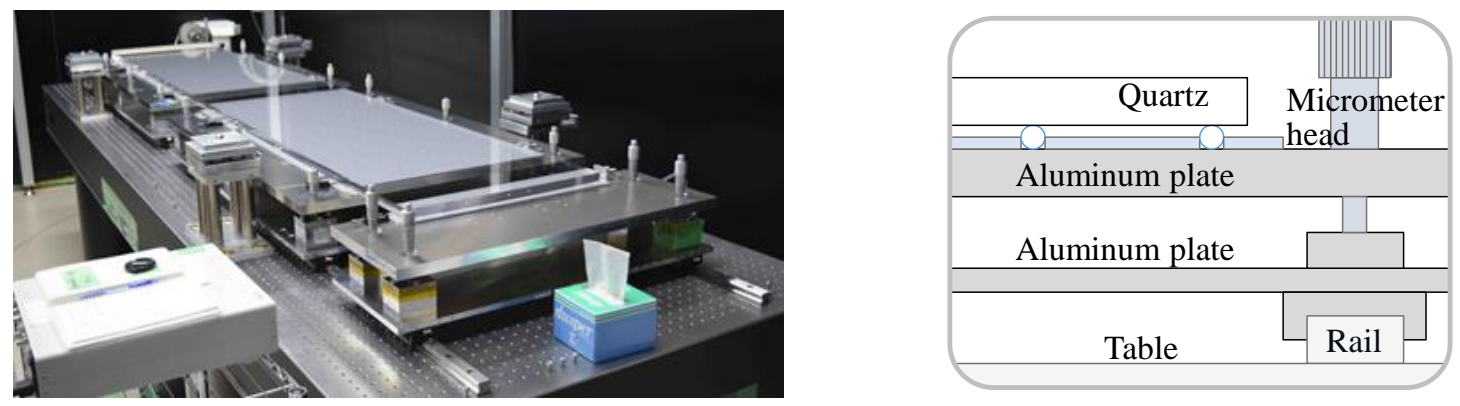

Figure 5: Alignment stages with the quartz optics (left) and a cross-sectional view of the stage (right).

the quartz optics in a clean room. A prism, two bars and another short bar with the mirror are put on special alignment stages as shown in Fig. 5. Each stage can be slid on the pair of the rails, and the tilt of the stage can be tuned with the micrometer heads. The procedures of the alignment and gluing are the followings: (1) Insert shims between the quartz joints to adjust the gap for glue. (2) Adjust surface positions of the quartz parts using a laser displacement sensor. (3) Adjust surface angles of the quartz parts using an autocollimator. (4) Iterate 2 and 3 several times until the required alignment is achieved. (5) Tape the quartz joints to make a dam and apply epoxy (EPOTEK 301-2) into the dam. The achieved horizontal and vertical deflection angle of the quartz joints was within \pm 40 and \pm 20 arcsec, respectively.

After the glue fully cures, the quartz optics is moved into a quartz bar box (QBB). As it is important to keep the quartz optics flat, the QBB needs high rigidity. On the other hand it is also important to have low mass for the experiment. Therefore the QBB is composed of aluminum honeycomb panels on the inner and outer faces for low mass and side rails made of monolithic aluminum from end to end of the QBB for high rigidity as shown in Fig. 6 (left). To increase the rigidity, the honeycomb panels are round shaped. The quartz optics is supported by PEEK buttons. The button height is tuned precisely with ring shims or bond thickness according to the optics alignment. The standard deviation of the height difference of the PEEK buttons on each honeycomb panel was kept within $0.02 \mathrm{~mm}$. For handling, the QBB is attached on a truss support called strong back as shown in Fig. 6 (right). With the strong back, the module sag can be kept
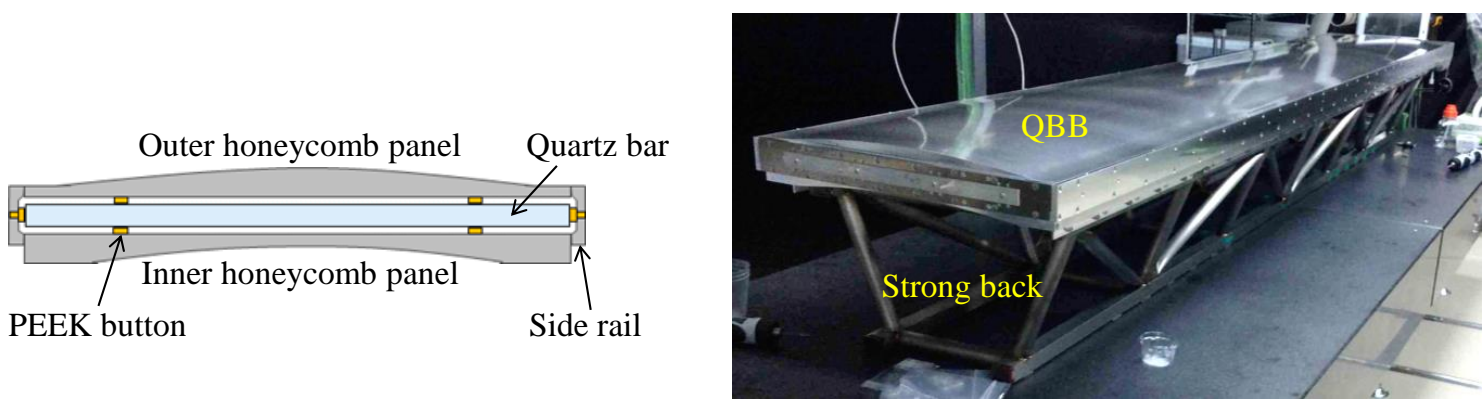

Figure 6: Cross-sectional view of the QBB (left) and the assembled TOP counter module with the strong back (right). 

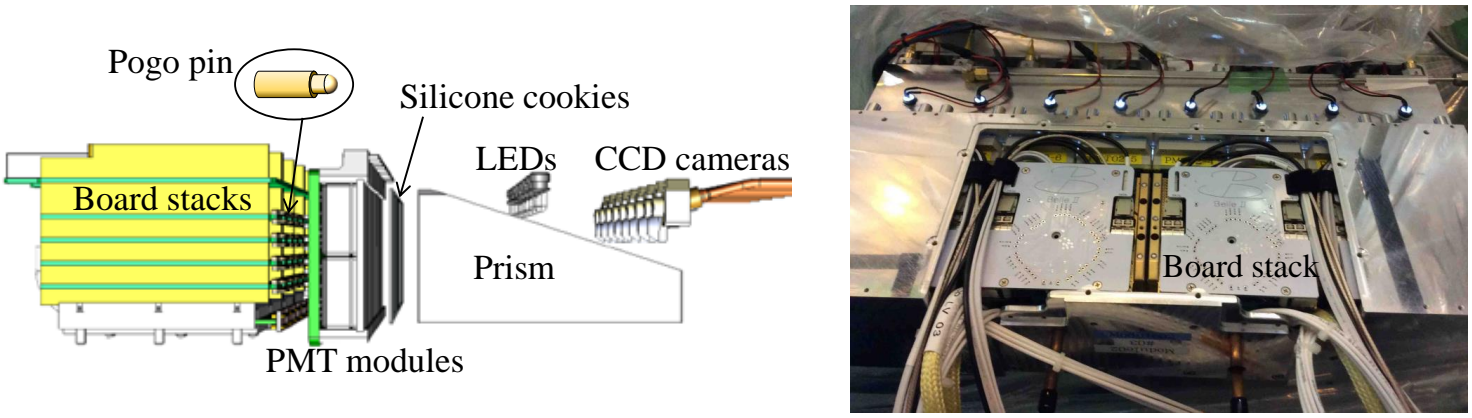

Figure 7: Schematic view of the readout part of the TOP counter (left) and a picture showing the installed board stacks, where only two of four board stacks can be seen from the opening (right).

below $0.5 \mathrm{~mm}$, which is necessary to securely support the quartz optics.

Lastly 32 MCP-PMTs and four readout board stacks are mounted on each TOP counter module from the opening of the enclosure as shown in Fig. 7. Four MCP-PMTs are assembled in a PMT module, and eight PMT modules are attached to the prism. In each PMT module the MCP-PMT windows are glued on a wavelength filter which cuts wavelengths below $340 \mathrm{~nm}$. The wavelength filter suppresses deterioration of the time resolution due to chromatic dispersion of the Cherenkov photons. To have a good optical contact between the wavelength filter and the prism as well as to make the PMT module replaceable, a soft cast silicone cookie is inserted. Just by pushing the silicone cookie with the PMT module, a bubble free contact can be made. It can be checked by the CCD cameras which view the photocathodes through the prism. The PMT module is interfaced to the board stack by spring-loaded electrical contacts (Pogo pins).

\subsection{Installation of the TOP counter}

The TOP counter modules were installed into the Belle II structure one-by-one. The first module was installed on 10 February 2016. A dedicated jig shown in Fig. 8 was built for the installation: The strong back was connected to the slider on the guide pipe; The module was slid into the barrel; Then it was rotated and approached to the position onto the sidewall. Some special
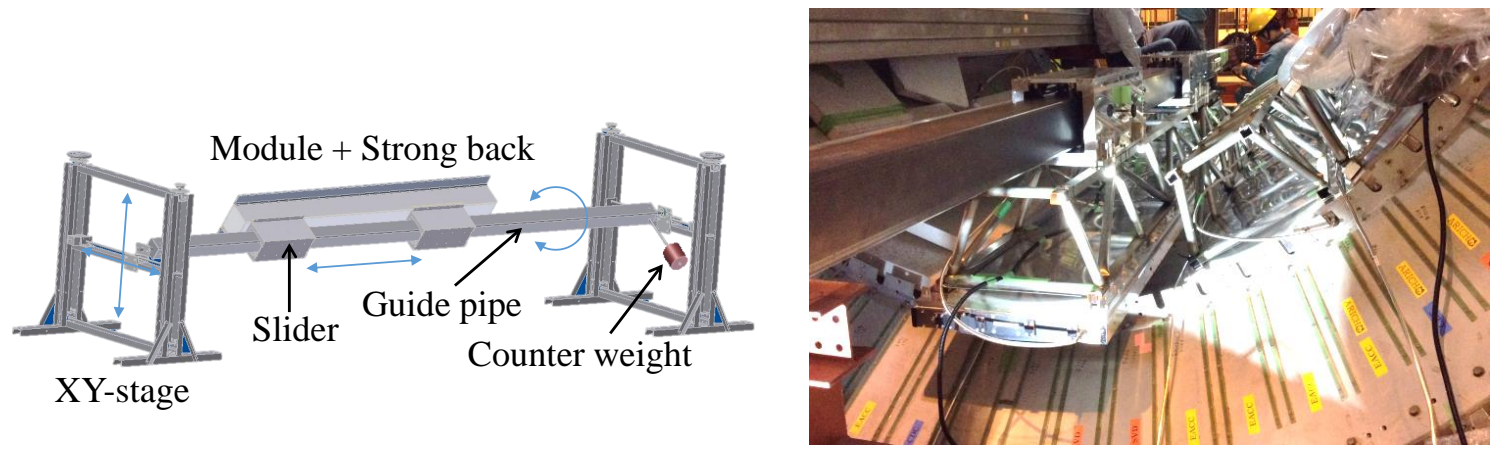

Figure 8: Schematic view of the installation jig (left) and a picture showing the third module being installed (right). 


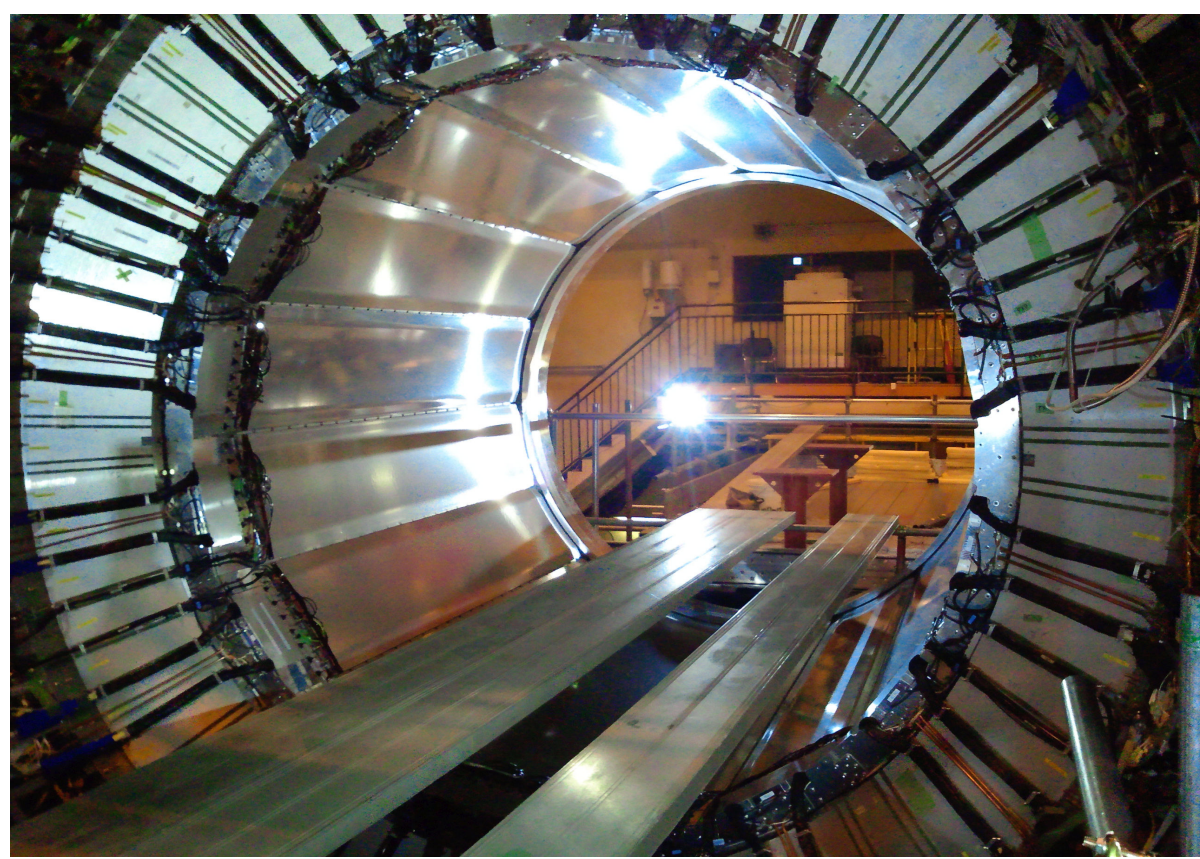

Figure 9: Belle II detector fully equipped with the 16 TOP counter modules.

cares were given to the design of this installation jig to keep the maximum sag below $0.5 \mathrm{~mm}$. The sag was monitored during the installation and it was confirmed to be less than $0.5 \mathrm{~mm}$.

The last sixteenth module was installed on 11 May 2016. Side-by-side modules were joined by $2.65 \mathrm{~m}$ long aluminum z-beams for structural integrity. Finally we came to the memorial day of 20 May 2016 when the strong backs were removed and the installation was completed successfully as shown in Fig. 9. The commissioning and calibration of the installed modules are ongoing toward the phase 2 operation for the SuperKEKB commissioning with the Belle II detector from January 2018 and the physics data taking from December 2018.

\section{Summary}

The TOP counter is one of the key devices in the Belle II experiment. After the long R\&D of more than a decade and the production and construction for two to three years, we finally succeeded in producing the 32 quartz bars and two spares as well as the other optics, producing the $512 \mathrm{MCP}$ PMTs and more than 60 spares, extending the MCP-PMT lifetime, assembling the 16 TOP counter modules and a spare, and installing them into the Belle II detector. That is one of the biggest milestones of the Belle II detector construction.

\section{Acknowledgement}

This work was supported by MEXT Grant-in-Aid for Scientific Research on Innovative Areas "Elucidation of New Hadrons with a Variety of Flavors" and JSPS Grant-in-Aid for Scientific Research (S) "Probing New Physics with Tau-Lepton" (No. 26220706). 


\section{References}

[1] Ed. A.J. Bevan, B. Golob, Th. Mannel, S. Prell, and B.D. Yabsley, Eur. Phys. J. C 74 (2014) 3026, SLAC-PUB-15968, KEK Preprint 2014-3.

[2] T. Abe, et al., KEK-REPORT-2010-1, 2010.

[3] Y. Ohnishi, et al., Prog. Theor. Exp. Phys. (2013) 2013 (3): 03A011.

[4] T. Ohshima, ICFA Instrumentation Bulletin 20 (2000) 10.

[5] M. Akatsu, et al., Nucl. Instr. and Meth. A 440 (2000) 124.

[6] T. Ohshima, Nucl. Instr. and Meth. A 453 (2000) 331.

[7] Y. Enari, et al., Nucl. Instr. and Meth. A 494 (2002) 430.

[8] E. Nakano, Nucl. Instr. and Meth. A 494 (2002) 402.

[9] M. Akatsu, et al., Nucl. Instr. and Meth. A 528 (2004) 763.

[10] Y. Enari, et al., Nucl. Instr. and Meth. A 547 (2005) 490.

[11] N. Kishimoto, et al., Nucl. Instr. and Meth. A 564 (2006) 204.

[12] K. Inami, et al., Nucl. Instr. and Meth. A 592 (2008) 247.

[13] T. Jinno, et al., Nucl. Instr. and Meth. A 629 (2011) 111.

[14] K. Matsuoka, et al., Nucl. Instr. and Meth. A 766 (2014) 148.

[15] K. Matsuoka, For the Belle II PID Group, Nucl. Instr. and Meth. A 732 (2013) 357.

[16] K. Matsuoka, For the Belle II PID group, PoS(TIPP2014)093.

[17] K. Matsuoka, et al., Nucl. Instr. and Meth. A, in press, http://dx.doi.org/10.1016/j.nima.2017.02.010.

[18] M. Andrew, PoS(TIPP2014)171. 